\title{
¿Estamos todos locos? El patrimonio de los no locos: excavaciones y estudios en el Hospital Moyano, Buenos Aires
}

Resumo: Em 1905 foi construído o mais importante laboratório de pesquisas neurológicas da América Latina, sob direção do alemão C. Jacob. Depois de sua morte, tudo ficou abandonado. Tentou-se recupará-lo como patrimônio, mas o estado de deterioração era tão grande que foi necessário fazer arqueologia no lugar em que estavam a biblioteca e as fotografias, escavando-se seus restos. Faz-se aqui uma análise do estado de deterioração da Ciência na Argentina e da pouca importância que se dá ao patromônio científico.

Abstract: In 1905, the largest laboratory in Latin America devoted to neurological investigation was built in Buenos Aires, under the direction of the German phisycian C. Jakob; after his passing, the premises were completely abandoned. Attempts were made to recover the structure for the national patrimony, but deterioration was so severe that archaeological excavations had to be conducted where once was the library and the photographic archives. An analysis is made about the deterioration of Science in Argentina and the little importance granted to its scientific heritage.

Palavras-chave: Patrimônio. Loucura. Arqueologia.

Key words: Patrimony. Madness. Archeology.

En la ciudad de Buenos Aires existe un conjunto hospitalario dedicado a los problemas mentales graves compuesto básicamente por dos unidades: una de hombres y otra de mujeres, habitualmente llamados "El Borda" y "El Moyano". Esos nombres son reducciones de sus verdaderas denominaciones; hasta hace

* Director del Centro de Arqueología Urbana (Universidad de Buenos Aires), Investigador Principal del Consejo Nacional de Ciencia y Tecnología, director del Area de Arqueología del Gobierno de la Ciudad de Buenos Aires.

Agradecimiento: el Dr. Jorge Colombo ha sido quien nos ha facilitado y hecho comprensible la información sobre la obra de C. Jakob en el campo neurobiológico.

Estudos Ibero-Americanos. PUCRS, v. XXXII, n. 2, p. 7-24, dezembro 2006 
unos años eran el Hospital Neuropsiquiátrico Tiburcio Borda (hombres) y Hospital Neuropsiquátrico Braulio Moyano (mujeres), hoy son sólo el Hospital Municipal Borda y el Moyano. En realidad cada uno de ellos está compuesto por innumerables pabellones y edificios dispersos por terrenos al sur de la ciudad, construidos desde la mitad del siglo XIX hasta la actualidad siguiendo el antiguo patrón hospitalario de pabellones separados entre sí para evitar los contagios. Quizás no sean los lugares más simpáticos para llevar a cabo estudios arqueológicos $\mathrm{o}$ patrimoniales ya que sus realidades son patéticas, a veces temibles y denigrantes para el ser humano, por la situación de desamparo, miseria y abandono.

El proyecto que se describe nació por la reacción de un grupo de profesionales, varios de ellos del Gobierno de la Ciudad de Buenos Aires, que plantearon duras críticas a lo que el mismo gobierno estaba haciendo: en el año 2002 y a través de sus organismos patrimoniales, al enterarse de la existencia de construcciones interesantes en el sitio, tuvieron la idea de usarlos para visitas turísticas con un sesgo histórico-patrimonial. Más allá de lo absurdo, rayando en lo patológico, de llevar gente de paseo a un manicomio para eso y peor a ese sitio destruido y que le pertenecía al mismo Gobierno que lo mostraba - y no críticamente por cierto a -, las autoridades, los visitantes y los guías caminaron por dos años sobre el derrumbe de lo que fueron los mejores laboratorios del país, sus bibliotecas desintegradas con libros y papeles en el piso, miles de negativos de vidrio aplastados y cientos de frascos de laboratorio sobre los que se colocaron tablones para caminar, destruyendo aun más todo. Abrieron el lugar al público que lo visitó sin que nadie moviera un dedo por salvar nada. Sólo importaba lo lóbrego, que era vendido al turismo ávido de emociones como un túnel antiguo en donde se "encontró tesoros y armas del gobernador y sus soldados", ${ }^{1}$ o que allí "Rosas escondía tesoros, para llevarlos desde allí al Riachuelo", ${ }^{2}$ absurdamente desde un aljibe moderno en una decoración de estilo neocolonial hecha seguramente hacia 1925.

Allí fue cuando se detuvo esto y se decidió hacer un estudio serio del conjunto, histórico, arqueológico - lo del sótano era realmente arqueología ya que era necesario excavar en el sedimento - y patrimonial; luego determinar su uso futuro. Los

1 EGGERS LAN, Margarita. Historia bajo las baldosas. Buenos Aires: Comisión de Preservación del Patrimonio Histórico-Cultural, Gobierno de la Ciudad, 2002, p. 97.

2 Idem, p. 95. 
estudios se comenzaron, la limpieza y protección también, ${ }^{3}$ y luego - nuevamente - todo quedó abandonado y liberado a la rapiña. Lo único que logró hacerse fue publicar la verdadera historia de las galerías y construcciones bajo el piso, ${ }^{4}$ y rescatar y limpiar un poco el sitio y los objetos que sobrevivieron antes de que de nuevo quede todo olvidado.

El proyecto de recuperación - aunque frustrado -, fue finalmente una iniciativa del mismo Gobierno de la Ciudad, aunque aceptando la necesidad de rescatar y estudiar antes de hacer otra cosa, como parte de un plan más amplio por recuperar la dignidad de esos sitios, no sólo desde la perspectiva médica y psicológica sino también porque encierran un alto valor patrimonial. ${ }^{5}$ Lo que era difícil de imaginar era la situación que encontramos y lo que ellos implica en cuanto a la destrucción de la ciencia y de las instituciones. En realidad nació como un rescate patrimonial en reacción a otro turístico descontrolado y terminó como una frustración, ${ }^{6}$ aunque estudiada arqueológicamente. Una experiencia más de la arqueología de una gran ciudad que constantemente obliga a replantear métodos, técnicas y hasta nuestra propia racionalidad como científicos ante la realidad que nos circunda.

\section{La historia}

El final del siglo XIX y los inicios del XX vieron la conformación de las profesiones y los campos científicos tal como los concebimos aun hoy. Hasta la década de 1860 la locura en Argentina era una curiosa mezcla de problemas policiales, resabios teológicos y una medicina incipiente que no explicaba casi nada más allá de separar, culturalmente por supuesto, lo considerado normal de lo pensado como anormal. Aun en la década de 1870 se publicaban tesis en la incipiente psiquiatría sobre temas como

3 Hospital Moyano: plan integral de recuperación y puesta en valor. Buenos Aires: Gobierno de la Ciudad, 2003.

4 SCHAVELZON, Daniel. Túneles de Buenos Aires. Buenos Aires: Editorial Sudamericana, 2005.

5 La Dirección General de Patrimonio logró intervenir en parte en la capilla, para restaurar la fachada.

6 El proyecto fue hecho por el Programa Bajo las Baldosas de la Comisión para la Preservación del Patrimonio Histórico Cultural del Gobierno de la Ciudad. El equipo de trabajo estuvo compuesto por Marcelo Weissel (coordinador), Patricia Frazzi, Graciela Torranzos, Eduardo Hernández, Rosario Aguilera, Ana Laura Spinetto, Enriqueta Ostrovich y Patricia Riádigos. 
Electrodiagnóstico, El cráneo y la locura, Espiritismo y locura, sus relaciones recíprocas. Pero en el mundo e incluso aquí la ciencia avanzaba rápidamente y el campo profesional que José Ingenieros describía como de gente que se interesaba en el mesmerismo o la frenología fue transformándose en conjuntos de especialistas que intentaban, a través de diferentes vías, acercarse a los problemas mentales e incluso a la mente misma y su funcionamiento. Nada estaba claro y obviamente la locura quedaba en terrenos que daban para todo, al menos hasta que dos grandes tendencias trataron de encontrar, y encontraron, vías de explicación: la neurobiológica y la psicológica. Es en ese contexto en que el país intentaba ganar un espacio en el mundo, en que los profesionales se encuadraron en instituciones, crearon laboratorios aceptando métodos y técnicas reconocidas y publicando sus experiencias, que se desarrolla esta historia.

Desde los inicios del siglo XIX hubo quienes trataron las afecciones mentales e hicieron lo que pudieron en su contexto histórico; lo sitios naturales eran las cárceles y los hospitales generales, no habiendo nada específico para ellos; sueltos o atados con cadenas, simplemente allí estaban. Quizás el primer escrito local fue del joven Diego Alcorta, que en 1827 se doctoró en medicina con una tesis sobre La manía, a la que siguió Martín García y su tesis sobre la epilepsia. Y aunque se pensó que en la época de Rosas no hubo trabajos de este tipo, el mismo Ingenieros cita la existencia de once tesis sobre temas mentales. Poco más tarde, para la mitad de siglo, se hizo habitual la relación entre los considerados locos y los médicos que lentamente se iban especializando en la materia y para finales de 1880 ya había más de cuarenta tesis escritas. Los temas que preocupaban están explícitos en esos títulos: El trépano cefálico, La vida y la muerte, El hipo, La afasia o Temperaturas del cerebro. Entre los que formaron estas primeras generaciones se destacaron José M. Ramos Mejía y luego Lucio Meléndez, quien en 1886 fue designado profesor de psiquiatría. La formación de todos ellos era ecléctica como lo era la ciencia de su tiempo y aunque pueda parecer muchas veces simple, violenta, agresiva y desconsiderada, la relación con los pacientes generaba posturas antitéticas. Ingenieros ${ }^{7}$ narra en relación a Meléndez que: "No existiendo en el Hospital de Hombres aula o anfiteatro para las lecciones teóricas, dábanse éstas en las habitaciones de los internos que estaban contiguas a

7 INGENIEROS, José. La locura en la Argentina. Buenos Aires: Cooperativa Editorial, 1920. 
los calabozos en el Patio de Dementes [...] y es verosímil suponer que esas circunstancias, coincidiendo con la de exponer sus lecciones en el patio de los orates, le indujesen a discurrir muchas veces sobre la miserable situación de los insanos que le rodeaban y sobre la naturaleza misma de las enfermedades mentales". De todas formas el mismo Ingenieros era líder de una línea muy dura, donde los límites con la criminología -su especialidad - y la psicología eran endebles; aunque la ideas de Lombrosio no tenían tanto pregón aquí nadie estaba demasiado lejos de ellas. De tal manera que muchas ideas finalmente servían para marginar, separar o reprimir todo disenso a un modelo social y cultural que estaba siendo impuesto. Así sigue narrando el origen de las ideas sobre la insanía mental: "El Hospital General de Hombres desde su fundación, a principios del siglo XIX fue en parte un depósito de alienados; su Patio de Dementes contenía más de la mitad de los enfermos hospitalizados. Es verosímil que esta circunstancia permitiese a los médicos de cierto ingenio, como el doctor Cosme Argerich, reflexionar sobre las ideas que comenzaban a romper las cadenas con que solía sujetarse a los infelices alienados".

Pero la enseñanza de la Patología Mental fue establecida en 1886 y puesta en manos del director del primer Hospicio de las Mercedes, Lucio Meléndez, quien a su vez fue quien determinó tanto el sistema de pabellones separados como la clasificación en agudos, crónicos, convalecientes, agitados, dementes, epilépticos, paralíticos, semiagitados, crónicos tranquilos y "los desaseados". El número de internados en 1880 llegaba a los 400 . Estos primeros hospitales implicaban pabellones amplios, entre jardines, con camas y baños; de la cárcel del cabildo a esto la distancia recorrida era enorme, aunque aun muy discutible en muchos sentidos.

En 1886 la Facultad de Medicina estableció el Curso de Clínica Psiquiátrica propuesto por el Dr. Cabred y puesto a cargo de Meléndez. En esos años haría su aporte sustancial al tema personalidades como Ricardo Sudnik y Andrés Llobet, todos ya trabajando en el tema de la conducción eléctrica del cerebro, el gran descubrimiento de su tiempo y en el cual nuestro país fue pionero absoluto. Al año siguiente bajo la administración del intendente Torcuato de Alvear se inauguró el Hospital de las Mercedes, cuyo primer director fue precisamente Menéndez y que hoy lleva como nombre, una vez separados en hombres y mujeres, de dos de sus discípulos: Moyano y Borda. En 1899 se creó el laboratorio de Anatomía Patológica a cargo de Cristofredo Jakob y 
en 1904 se demolió el edificio del asilo para completar las edificaciones que aun existen. En 1888 se había establecido la cátedra de enfermedades nerviosas confiándose su desempeño al doctor José M. Ramos Mejía. Lentamente se iban separando la psicología de la psiquiatría, la medicina cerebral y luego los estudios biológicos y anatómicos; asimismo se iba completando el traspaso, que llevó mucho tiempo, de la medicina y la salud que fueron primero campos de la religión, luego de la beneficencia, al Estado Nacional y finalmente al municipal. Para la llegada desde Alemania de Cristofredo Jakob, o más precisamente el hecho de su llegada, se debió a la existencia de un campo de estudio consolidado dentro de la ciencia, nacional e internacional.

\section{Cristofredo Jakob en Argentina}

La historia de Cristofredo (Christfried) Jakob está ligada en forma indisoluble al desarrollo de la neurociencia en Argentina. $\mathrm{Su}$ aporte al campo de la neuroanatomía y neurohistología comparadas y las funciones cerebrales está bien a la altura de otros insignes nombres de ese periodo de la investigación cerebral como Ramón y Cajal o Golgi. Sin embargo, la bibliografía no se ha ocupado de citar su obra en proporción a ello - decidió actuar en un país del tercer Mundo al fin de cuentas -, plasmada en unas treinta monografías y más de doscientos artículos ricamente ilustrados con estudios microscópicos, una épica de su tiempo.

Jakob fue invitado a venir de Europa durante el gobierno del general Roca, al igual que un numerosos grupo de científicos en todas las ramas del conocimiento y las artes. La invitación en este caso provino del doctor Domingo Cabred quien estaba buscando allí al director de un nuevo laboratorio de clínica neuropsiquiátrica en el Hospicio de las Mercedes. En esos años el país, o al menos su clase dirigente, tenía muy en claro cual era el camino de desarrollo que querían y no se dudó en gastar lo que fuera necesario en ello, de allí el traer a este joven prometedor alemán. Jakob era un médico psiquiatra dedicado con éxito desde el inicio de su carrera a la neurobiología; había nacido en Alemania en 1866 y murió en Buenos Aires en 1956; fue maestro de varias generaciones de biólogos, psiquiatras y neurobiólogos argentinos, no casualmente los más destacados y los que establecieron la orientación anátomopatologica en la disciplina, 
lo que hicieron en ese laboratorio. Su primer gran obra fue un Atlas del sistema nervioso. ${ }^{8}$ En 1899 llegó a la Argentina para dirigir el Laboratorio de Anatomía Patológica que funcionaba en la Cátedra de Clínica Psiquiátrica.

Pero obviamente su estadía local generó enormes conflictos de poder y prestigio, en especial con Llobet, lo que se acrecentaba porque Jakob era luterano y su vecino, competidor y colega era ferviente católico, mientras que Ramos Mejía era un activo Masón como gran parte de los científicos y políticos de avanzada de su tiempo. ${ }^{9}$ El Laboratorio sería por mucho tiempo su lugar de trabajo hasta que tuvo que renunciar y regresó a Alemania en 1910 publicando allí un importante libro sobre el cerebro humano; también tradujo al alemán un estudio sobre la neurología de los mamíferos que había escrito junto a Clemente Onelli en el Jardín Zoológico de Buenos Aires y que publicó en 1913.10

Sus libros generaron un impacto tan fuerte en el medio internacional que fue invitado a regresar de inmediato, por lo que volvió para hacerse cargo de las cátedras de biología tanto en la universidad de Buenos Aires como la más reciente de La Plata. Por entonces compuso su obra principal, la Folia Neurobiológica Argentina, que consta de varios tomos. La obra trata acerca de la estructura del tejido nervioso en el hombre y en los animales. Estos estudios demostraban la importancia de la arquitectura del cerebro para la comprensión de los problemas psiquiátricos y neurológicos. Sus contribuciones al desarrollo de los conceptos de organización de la corteza cerebral eran de

8 JAKOB, Cristofredo. Atlas der Gesunden und Kranken Nervensystems nebst Grundriss der Anatomie, Pathologie und Therapie desselben. München: Verlag von J. Lehmann, 1895 (segunda edición: 1899); y Atlas der Klinischen Untersuchungsmethoden nebst Grundriss der Klinischen Diagnostik und der speziellen Pathologie und Therapie der inneren Krankheiten. München: Verlag von F. Lehmann, 1897.

9 Estos conflictos se analizan en los artículos de CROCCO, Mario. El contexto histórico y los descubrimientos de Alberto Alberti (1883) en las localizaciones cerebrales. $\mathrm{La}$ Semana Médica (5 de abril), p. 219-230, 1986; CROCCO, Mario y N. C. Contreras, Andrés F. Llobet y Cristofredo Jakob en la primigenia neurobiología argentina, La Semana Médica (5 de mayo), p. 317-330, 1986; CROCCO, Mario F. Oscuridades, enigmas y el aporte fundamental de Ricardo Sudnik (1880-84) y el origen de la neurobiología argentina, La Semana Médica (12 de marzo), p. 141-151, 1987.

10 JAKOB, Cristofredo. Das Menschenhirn (eine Studie über den Aufbau und die Bedeutung seiner Grauen und Rinde). Einführung in den Organisationsplan der Menschlichen Zentralnervensystems. Munchen: J. F. Lehmann's, 1911; Vom Tierhirn zum Menschenhirn. Einführung in die Geschichte der Hirnrinde. Munchen: J. F. Lehmann, 1911. 
avanzada y anticiparon ponencias que más tarde harían otros autores, muchas veces sin recibir el reconocimiento que correspondía. Es por eso importante señalar algunos ejemplos de lo creativo de varias de sus propuestas, como las expresadas en su Folia Neurobiologica:11 una se refiere a la organización vertical de la citoarquitectura de la corteza cerebral, que fuera descripta e ilustrada por Jakob y Clemente Onelli en 1913 y luego formalizada por Lorente de Nó alrededor del año 1931, y a partir de la década del 1950 por los proponentes de la organización columnar de la corteza cerebral hasta constituirse en una representación funcional al desarrollo de conceptos modernos en neurociencia cognitiva. La segunda fue la conceptualización de un llamado cerebro visceral, anticipándose nuevamente al resto del mundo incluyendo la propuesta de que el cerebro humano incorporaba niveles de organización ancestrales a la evolución de los primates. De tal manera anticipó a quienes mucho más tarde vislumbraron la organización cerebral como compuesta por tres niveles filogenéticos.

Elaboró un Tratado de biología general y especial que se publicó en 1915 en cuatro tomos, en los cuales tomó en consideración las características locales planteando que los problemas mentales no eran iguales en todas partes. La elaboración de esta obra se enmarcaba en una idea más general que Jakob promovía: la de la enseñanza de la biología ya no como una ciencia ocupada de la taxonomía y la descripción sino como la ciencia que debía reflejar la realidad circundante. En 1919 se hizo cargo de la Cátedra de Anatomía Descriptiva de la Facultad de Medicina a la que renunció por problemas políticos. Fue un entusiasta explorador del territorio y de vastas regiones de Perú, Bolivia y Chile, visitó zonas poco conocidas y realizó evaluaciones del potencial productivo de diversas regiones. Muchos de estos viajes fueron relatados en artículos que publicó en la Revista Geográfica Americana y en los Anales de la Sociedad Científica Argentina. Mantuvo su labor formativa y científica en la Cátedra de Anatomía Patológica hasta 1953 y falleció dos años después. Actualmente, varios institutos neuropsiquiátricos del país llevan su nombre.

11 La Folia Neurobiológica Argentina se inicia con "El cerebro humano" (tomo I de 1939) y continúa hasta "Documenta biofilosófica" (tomo V, 1946), además de tres volúmenes del Atlas, editado por Aniceto López, Buenos Aires. 
El edificio del que más adelante hablaremos y que genera este estudio fue el segundo laboratorio de investigación anatomopatológica. Corresponde a la segunda etapa de su producción científica, la primera fue en el laboratorio del actual Hospital Borda; fundó allí un museo neurobiológico que es lo que hoy persiste y es considerado como casi único dado que los europeos se destruyeron en las guerras del siglo XX. En él y tal como lo entendía Jakob, la anatomía comparada del sistema nervioso abarca desde los animales al hombre y precisamente por eso fue que junto al director del zoológico Clemente Onelli, investigaron toda la serie de cerebros animales.12 La producción científico en su campo específico es de cerca de doscientos estudios imposibles de citar completos. ${ }^{13}$

Entre otras actividades colaboró con la publicación de la Revista del Jardín Zoológico, ${ }^{14}$ uniendo dichos artículos en su obra Biología general y especial. Buena parte del material fotográfico de ésta obra se encuentra en los preparados dejados por él en el Museo, otra parte es la de cuya destrucción hablaremos. También de acuerdo a su tiempo era un naturalista completo con amplios conocimientos antropológicos, paleontológicos, obviamente de zoología por lo que hizo estudios con material proveniente del Museo de La Plata. ${ }^{5}$ En la madurez hizo y publicó sus pensamientos y sobre filosofía; 16 en la juventud había escrito

12 JAKOB, Cristofredo y ONELLI, Clemente. Atlas del cerebro de los mamíferos de la República Argentina. Congreso Científico Internacional Americano. Buenos Aires: Imprenta de Guillermo Kraft, 1913.

13 Una buena bibliografía casi completa puede verse en:

$<$ http:/ / electroneubio.secyt.gov.ar>, además existen allí varias biografías y estudios técnicos sobre su obra.

14 JAKOB, Cristofredo. Diferencia entre inteligencia humana y animal. Revista del Jardín Zoológico de Buenos Aires. 1913; El lenguaje de los animales. Revista del Jardín Zoológico de Buenos Aires, 1914; El cultivo artificial del órgano del pensamiento. Revista del Jardin Zoológico de Buenos Aires, 1914; Un gibón y un chimpancé autopsiados. Revista del Jardín Zoológico de Buenos Aires, 1914; La locura en los animales. Revista del Jardín Zoológico de Buenos Aires, 1915; Hallazgo de pigmento amarillo y negro en las células nerviosas de un mono adulto. Revista del Jardín Zoológico de Buenos Aires, 1915.

15 JAKOB, Cristofredo. Contribución al estudio del cerebro de los indios. Anales del Museo de La Plata. 1904; Sobre cerebros fósiles de la fauna argentina. Actas del XVII Congreso Internacional de Americanistas. Buenos Aires, 1912; Atlas y Axis, contribución a su biología con una advertencia respecto del "Atlas de Monte Hermoso", Revista de la Asociación Médica Argentina. Vol. I, 1940.

16 JAKOB, Cristofredo. La biología en el sistema de las ciencias filosóficas y naturales. Publicación de la Universidad de Buenos Aires. Imprenta Coni Hnos, 1913; Del tropismo a la teoría general de la relatividad. Humanidades. T. III, 1922; Problemas actuales de psiquiatría general y sus relaciones con las ciencias sociales y jurídicas. Revista del Círculo Médico Argentino, 1916; Localización del alma. El libro vol. 1 a 5, 1904-08. 
manuales de biología para uso escolar ${ }^{17}$ y publicó hasta estudios biogeográficos. ${ }^{18}$

\section{EI Pabellón Jacob en el Hospital Moyano}

La obra de Jakob por la ciencia quedó guardada en el sitio donde se produjo, primero en el pabellón del Hospital Borda y luego en el del Moyano. Tras su renuncia en 1953, ya siendo un hombre muy mayor, todo quedó abandonado o con uso esporádico. Es cierto que el mundo había cambiado, también la ciencia, y era complejo sostener estos enormes edificios que necesitaban mantenimiento constante y que eran entendidos como cátedras, museos, laboratorios, lugar de autopsias y múltiples usos más. De sus laboratorios el del Borda se mantiene, es Monumento Histórico Nacional ${ }^{19}$ y sus condiciones son modestas, con equipamiento antiguo - la mayor parte es de la década de 1950 con un sector destinado a museo; el del Botánico fue demolido en el año 2000 sin objetivo definido.

El ahora llamado Pabellón Jackob en el Moyano, construido posiblemente en 1905, es un bloque aislado al fondo de los grandes terrenos del conjunto hospitalario, construido en la típica arquitectura estatal de su tiempo, monumental, afrancesado, poco funcional, muy impresionante a la vista, que debió ser perfecto para dar la imagen de lo que se quería dar: un pabellón hospitalario dedicado a la investigación, pero que debió ser en extremo complejo y caro de mantener en uso con mucho personal para ello. Es por cierto muy similar, aunque en escala más grande, al construido años antes en el Hospital Borda. El plano muestra

Buenos Aires, hay edición en forma de libro; El espíritu de la música en la filosofía pre y postkantiana. Buenos Aires: Humanidades. T. XIII, 1926; La definición científica de la vida. Buenos Aires: Verbum s.d., 1935; Descartes en la Biología. Publicación de la Facultad de Filosofía y Letras, 1937. Buenos Aires; La Psicología de Descartes a través de tres siglos. Anales del Instituto de Psicología. T. II, 1938; El significado de la obra de Ramón y Cajal en la filosofía de lo orgánico. Humanidades. T. XXVI. 1938; El cerebro humano, su significación filosófica; Ensayo de un programa Psico-bio-metafísico después de cincuenta años de dedicación neurobiológica. Revista de Neurología de Buenos Aires. Vol. X., n. 2. 1945; El origen de la conciencia. Anales de Psicología Buenos Aires, 1945.

17 JAKOB, Cristofredo, Tratado de Biología General y Especial para el uso de la enseñanza elemental, secundaria y superior en la República Argentina. Buenos Aires, 1915-18, 2 volúmenes. Imprenta de G. Kraft; Sobre la enseñanza de las ciencias biológicas en la escuela primaria y secundaria. Humanidades. Vol. XVI, 1927, Buenos Aires.

18 JAKOB, Cristofredo. Alrededor del Tronador; excursiones biogeográficas en la Suiza Argentina. Revista Geográfica Americana. Año III, n. 28, 1936.

19 Declarado el 15 de abril 1999. 
una solución arquitectónica clara: la planta baja, elevada como corresponde en medio nivel, tiene una gran sala de disecciones que es aula y auditorio a la vez, con graderías elevadas para ver la mesa de operaciones en el centro la que está cubierta por un grueso mármol. Aprovechando la estructura de madera hay complejos muebles de época que servían para guardar millones de preparados histológicos de cortes cerebrales en cajas especiales, grandes frascos cuadrados de vidrio para guardar cerebros en formol y una decoración en relación con la funcionalidad del sitio. Luego habían diversas oficinas y la lógica morgue con sus heladeras y piletas de formol, todo lo que aun sigue en uso, con tétricas historias en especial de los tiempos de las dictaduras militares. El conjunto de lo existente en este piso parece detenido en el tiempo, salvo por el busto de Jakob que mira sin ver el paso del tiempo y de las gentes; placas de mármol con inscripciones dan el tono de sitio sagrado dedicado a la ciencia: "Aquí los muertos le hablan a los vivos", dice en latín una enorme placa grabada que posiblemente hoy ya nadie entienda.

$\mathrm{El}$ edificio tiene una escalera interior que permite descender al nivel semi-subterráneo donde estaban los laboratorios, farmacia, biblioteca, fotografía y hornos para cremar cadáveres. Era el sitio más usado por quienes allí trabajaban y al parecer en algún momento - creemos hacia la época en que Jakob deja todo el tema -, quedó abandonado. El superior siguió en uso para dar clases en el aula-auditorio y la morgue para la policía; qué pasaba o qué se hacía en el resto de ese enorme pabellón olvidado, nadie lo sabrá jamás. La parte de abajo tenía numerosos ambientes ya que Jakob invitaba no sólo a alumnos y docentes sino a expertos de otros países a colaborar con él, por lo que en su tiempo habían laboratorios menores para alemanes, franceses, ingleses y quienes vinieran a este centro mundialmente conocido en la materia.

El problema principal que tenía el edificio era constructivo: pese a la calidad de todos los materiales el sistema de iluminación natural elegido sólo servía mientras se lo mantenía con mucha mano de obra: las ventanas estaban mitad afuera y mitad debajo del nivel externo, en una especie de caja con piso, que si bien facilitaba en mucho la entrada de luz, también entraba el agua de lluvia y la tierra. Es cierto que en origen tenían un sistema de desague pero con el menor abandono este se taponó y por allí entró agua y tierra y gatos y gente y basura, inundándose a la primera lluvia fuerte. Y así siguió por medio siglo. Efectivamente eso es lo que entendemos que pasó: comenzó a llenarse de agua que pudrió los muebles de madera y oxidó los de metal, todo cayó al piso incluyendo las bibliotecas y 
sus libros, los estantes de fotografías de vidrio, las cámaras fotográficas de fuelle, los medicamentos... Y se fue formando una pasta, abonada por ratas y gatos y seguramente humanos, que cubrió los pisos con diferentes tenores de humedad según la temporada del año. Sin ninguna duda jamás habíamos visto un estrato arqueológico de estas características, capaz no sólo de dar información del pasado sino también de sumir en la depresión a quien siquiera lo veía. Si la planta del edificio mide unos 20 por 50 metros, pensemos que ese sedimento se extendía por cien metros cuadrados con alturas de hasta dos metros.

\section{El contexto arqueológico}

Cuando un arqueólogo habla de su "sitio" y del "sedimento" que lo compone uno tiende a imaginar un lugar abierto y un piso de tierra donde excavar. Aquí el lugar era un sótano que tenía un piso de cemento y el sedimento era lo que estaba arriba de ese nivel, no abajo, lo cual no sólo presentaba problemas técnicos serios, sino también conceptuales. ¿Existe una teoría o una metodología para este tipo de intervenciones? ¿La más osada aventura del pensamiento de quienes estudiaron las alteraciones de un sitio pasó por este tipo de casos? Creo que podemos afirmar que no.

El sedimento estaba en todos los casos sobre un contrapiso alisado de cemento; sobre él había, según las habitaciones, desde una delgada capa de tierra y fragmentos de vidrios de ventanas con objetos esporádicos muy recientes, hasta más de un metro de una pasta de papel, tierra, madera podrida, hierro oxidado, vidrio de todo tipo fragmentado hasta milímetros por pisoteo, productos medicinales y farmacéuticos y sus envases, basura moderna reciente (botellas, plástico, corchos, bolsas, preservativos, gatos muertos, entre otras cosas), cientos de frascos vacíos y llenos de suero y, para compactar todo, tablones de madera puestos por el Gobierno de la Ciudad para circular por encima. En algunas habitaciones el relleno era básicamente de cientos de miles de fragmentos de negativos de vidrio quebrados, mezclados con barro y desechos varios, pisoteados hasta compactar todo en una masa casi sólida y continua. En el laboratorio fotográfico, donde la maquinaria original estaba aun en pie aunque destruida por el óxido, las cámaras y sus trípodes; como nada fue tocado la madera se pudrió, cayeron las cámaras donde estaban y quedaron en el piso los fragmentos metálicos oxidados y los lentes, todo en su posición original. 


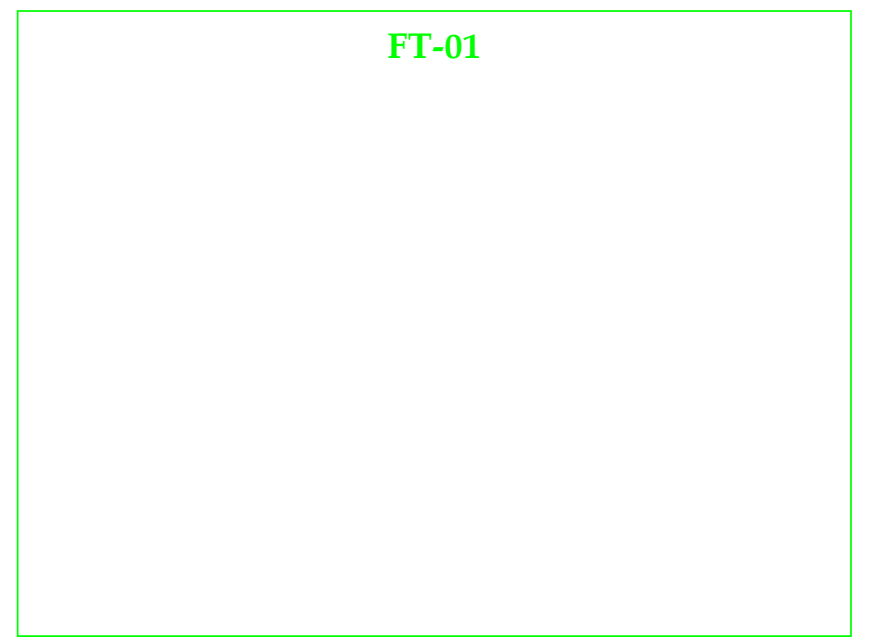

Cabe destacar que la observación de las placas negativas de vidrio permitió entender que se trataba de dos grandes conjuntos: uno de fotografías de las internas, y otro conjunto de cortes histológicos. El estado de deterioro que hizo que la emulsión pegara una con otra, lo que salvó muchas de ser aplastadas pero hará en extremo complejo su restauración, de ser siquiera factible poder hacerlo. Los frascos medicinales y farmacéuticos aun contienen productos en su interior, los que al moverlos producen olores y coloraciones nauseabundas y cuyo control de seguridad fue y será complejo. Los objetos de laboratorio, como probetas y tubos de ensayo, por lo general estaban absolutamente destruidos dada su extrema fragilidad.

\section{La construcción de hipótesis y objetivos}

Tras las primeras visitas, al entender lo que se estaba enfrentando y en función de una operación de rescate para el Gobierno de la Ciudad que exigía rapidez de respuesta para mantener el lugar visitable, se establecieron los siguientes objetivos: 1) recuperar todos los objetos materiales que pudieran ser parte de futuras exhibiciones, que mostraran tanto lo que fue como el proceso de degradación misma; 2) limpiar el lugar para poder acceder a él con seguridad, en especial de productos tóxicos químicos y farmacéuticos; 3) establecer un proyecto patrimonial para el lugar; 4) tratar de entender, desde una mirada arqueo- 
lógica-histórica, el proceso de transformación del sitio desde su forma de uso original hasta el presente; 5) tratar de entender qué había pasado allí y cómo era factible que lo que fue un famoso laboratorio internacional haya terminado de esa manera. En función de ello y entendiendo que se trataba de una operación de rescate con pocos recursos y tiempo, en un entorno altamente tóxico, se optó por tres formas de acción: 1) limpieza simple de todos los ambientes que quedaron vacíos desde antiguo; 2) recuperar los materiales identificables: muebles o parte de ellos, todo lo farmacéutico que estuviera en condiciones de ser exhibido o restaurado, los objetos constructivos para restaurar el lugar (puertas, marcos, ventanas, baldosas); 3) recuperar con especial cuidado los negativos de vidrio enteros o parcialmente rotos para una posible restauración y guardado; 4) trabajo arqueológico en los ambientes en que había sedimento acumulado no distinguible.

\section{Los trabajos realizados}

El estado general del sitio es, para su arquitectura, malo aunque la construcción sigue en pie; por lo tanto con una limpieza, arreglos generales de carpinterías, techos y muros de azulejos desprendidos, el lugar puede ser visitado sin problemas, aunque cerrando los conductos a otro subsuelo que conducía el agua hacia el exterior. No había iluminación y se hizo una instalación provisoria, la que por su precariedad tenía su propio encanto para el visitante. Luego se hizo la limpieza y el retiro de escombro, recuperación de maderas provenientes de las carpinterías, incluidas puertas que habían sido desarmadas y estaban listas para ser robadas, la recuperación de muebles y estanterías los que aunque muy deteriorados permitieron rearmar parte de la vieja farmacia, la recuperación de unos 2.500 frascos de vidrio de diversos tamaños: botellas, recipientes para sueros o frascos con tapa esmerilada entre tantos otros, recuperación de unos trescientos recipientes de lata con tapa, unas cien damajuanas antiguas de las que la mitad tenía aun su caja o canasto protector y la instalación de todo eso en ambientes protegidos. Con los negativos de vidrio, que llenaban dos habitaciones completas, se logró extraer unos 4.500 enteros o poco fragmentados, mientras que cientos de miles de pequeños fragmentos que formaban parte del sedimento no fue posible protegerlos por la decisión política de no usar mayor tiempo. Fueron ordenados y guardados en un sitio cerrado. Todo este trabajo fue hecho por las restauradoras y 
conservadoras del equipo, todos voluntarias, con serios riesgos para su salud, incluso siendo necesario dejar el trabajo por varios días ante la rotura de frascos que despedían olores o humaredas no identificables. No fue más que un primer trabajo elemental, se suponía que a la brevedad habría acciones de preservación serias y sistemáticas.

En forma paralela a estos trabajos se hizo un estudio del sistema de galerías subterráneas del cercano Pabellón Charcot para explicar racionalmente de qué se trataba y evitar que se siguiera publicando información no sólo errada sino históricamente absurda. 


\section{El sedimento arqueológico}

Los sectores que pudieron ser excavados arqueológicamente presentaron un sedimento compacto, altamente pisoteado en fecha reciente, ayudado por la concentración de agua cada lluvia. La estratigrafía general mostró un primer nivel sobre el piso original formado por tierra y vidrios provenientes de las grandes ventanas; lo interpretamos como la rotura - natural o intencional de los cerramientos y el polvo que entró por ellos con el tiempo. Un segundo estrato está conformado por madera de muebles, concretamente estanterías de la biblioteca que debió existir contra los muros. Este nivel tiene como sedimento una pasta de papel proveniente de libros y hojas escritas totalmente destruidas mezcladas con la paste de la madera y tierra en casi $35 \mathrm{~cm}$ de altura. Luego hay alternados estratos de tierra apisonada, objetos recientes, muchas botellas de vino y damajuanas y sus envases de madera, fragmentos de ladrillos y de frascos de medicina y farmacia. Básicamente se trata de un sedimento formado por pasta de libros destruidos y placas de vidrio pulverizadas con los restos de sus muebles. Un aporte importante al caos de este conjunto fueron los grandes frascos cuadrados de vidrio en que se colocaban en exhibición los cerebros $\mathrm{u}$ otros tejidos; muchos de ellos se salvaron al caer por su propia resistencia aunque otros dejaron sus fragmentos - y posiblemente su contenido - en el sedimento.

En forma sintética entendemos que lo sucedido es que tras la rotura de los vidrios de las ventanas y el consecuente ingreso de agua se pudrieron las bibliotecas y estanterías de madera, se cayeron mezclando los libros y papeles creando una mezcla ahora rígida pero en la cual se distinguen los componentes, luego ingresó gente que caminó por encima e hizo uso del lugar dejando botellas y materia fecal, posiblemente internos del hospicio; entre ello hubo varios gatos muertos en diferente estado de putrefacción. La presencia de una capa superior de frascos de medicinas y material de farmacia lo entendemos como parte del saqueo más reciente de objetos con el afán de venderlos a coleccionistas y anticuarios; es posible que el sector de la farmacia haya quedado mejor cerrado y sólo cuando fue encontrado se quiso aprovechar lo que allí había, dejando frascos y probetas por todos lados. La forma en que afectaron los productos químicos y el formol de damajuanas y cajas de vidrio en este conjunto, resulta imposible de determinar, pero debe haber ayudado a darle consistencia. Si además se deshicieron allí fragmentos de tejidos humanos, incluso es mejor 
no pensarlo aunque suene poco científico. Los centenares de grandes damajuanas de formol, sistemáticamente abiertas y cuyos corchos están en la zona, lo interpretamos no como simple abandono sino que fueron usadas posiblemente para drogarse; en situaciones límites como las que hay que un hospital mental este tipo de productos suele usarse con ese fin según información obtenida del personal médico del lugar.

Mención aparte merece lo ya dicho de los miles de negativos de vidrio que aparecen por todas partes y muy pulverizados, mucho más que rotos, aunque en dos sitios del edificio los hay en gran cantidad, tanto enteros o poco quebrados, incluido una biblioteca que no llegó a caerse completa y en cuyos cajones hallamos cientos de ellos; es decir que en algunos lugares sólo los estantes se vencieron y cayeron directamente al piso, en otros fueron arrojados y luego pisados una y otra vez. ${ }^{20}$

Si bien la interpretación de la excavación coincide y se hace coherente con todo lo sucedido en el sitio, es decir con su contexto - obvio en muchos sentidos -, es significativo en términos de tratar de entender el proceso de destrucción de un legado científico y patrimonial de inmenso valor por simple desidia, ineficacia, burocracia o desconocimiento.

\section{Conclusiones}

En el Pabellón Jakob del Hospital Borda, debajo del acceso y su cartel de grandes letras hecho en 1899, alguien escribió con otras aun más grandes letras verdes "Estamos todos locos". Creo que es la mejor conclusión posible y la tomaron los mismos internos, o alguno de ellos. Lo que sucedió con la memoria de Jakob, con ese excepcional edificio en el Hospital Moyano, con la investigación científica argentina, con un laboratorio de primer nivel mundial y totalmente equipado, parece imposible. Pero aunque aceptemos lo absurdo de dejar abandonado decenios de trabajo de excepcional calidad científica y culpemos como hacemos a las dictaduras, a la primacía de lo psicológico sobre cualquier otra explicación de los fenómenos mentales, a la falta de dinero o a lo que sea, nada explica lo posterior: la destrucción a mansalva de ese patrimonio, el caminarle por encima, organizar visitas turísticas para lucir nuestra desidia; y la imposibilidad de que

20 No se autorizó la conservación ni restauración de esos negativos, sólo se logró retirar una muestra que ha sido guardada en el Centro de Arqueología Urbana (FADU, UBA), el resto quedó allí lamentablemente; su estado actual lo desconocemos. 
después de rescatado oficialmente, aunque lo haya sido con esfuerzo voluntario, y publicado y puesto en internet para lucir lo hecho, todo vuelva a quedar abandonado al año siguiente.

Es cierto que esto es poco arqueológico, pero nos lleva al punto central: cómo podemos hacer arqueología ascéptica en la realidad social que nos ha tocado vivir. Porque al fin de cuentas el excavar "un sedimento formado por pasta de libros destruidos y antiguos negativos fotográficos de vidrio pulverizados" no parece resultar otra cosa que el ya citado "Estamos todos locos".

\section{Bibliografía}

EGGERS LAN, Margarita. Historia bajo las baldosas. Buenos Aires: Comisión de Preservación del Patrimonio Histórico-Cultural, Gobierno de la Ciudad, 2002.

DIRECCIÓN GENERAL DE PATRIMONIO. Hospital Moyano: plan integral de recuperación y puesta en valor. Buenos Aires: Gobierno de la Ciudad, 2003.

INGENIEROS, José. La locura en la Argentina. Buenos Aires: Cooperativa Editorial Limitada, 1920.

SCHÁVELZON, Daniel. Túneles de Buenos Aires. Buenos Aires: Editorial Sudamericama, 2005. 